See discussions, stats, and author profiles for this publication at: https://www.researchgate.net/publication/303098767

\title{
Traveling Between the Earth-Moon Lagrangian Points and the Earth
}

Conference Paper · May 2016

DOI: $10.2514 / 6.2016-2558$

CITATIONS

2

4 authors, including:

Geraldo Magela Couto Oliveira

Centro Federal de Educação Tecnológica de Minas Gerais

9 PUBLICATIONS 18 CITATIONS

SEE PROFILE

Diogo Merguizo Sanchez

National Institute for Space Research, Brazil

39 PUBLICATIONS 109 CITATIONS

SEE PROFILE

Some of the authors of this publication are also working on these related projects:

Project Doctorate Degree View project
READS

395

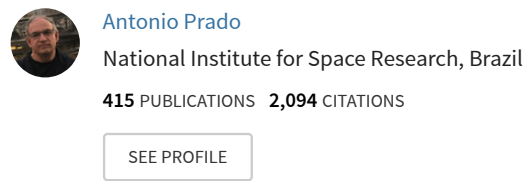




\title{
Traveling Between the Earth-Moon Lagrangian Points and the Earth
}

\author{
Geraldo Magela Couto Oliveira ${ }^{1}$ \\ Federal Center for Technological Education of Minas Gerais - CEFET-MG, Contagem, Minas Gerais, 32.210-160, Brazil \\ Antonio Fernando Bertachini de Almeida Prado ${ }^{2}$ and Diogo Merguizo Sanchez ${ }^{3}$ \\ National Institute for Space Research - INPE, São José dos Campos, São Paulo, 12.227-010, Brazil \\ and \\ Vivian Martins Gomes ${ }^{4}$ \\ São Paulo State University - FEG/UNESP, Guaratinguetá, São Paulo, 12.516-410, Brazil
}

\begin{abstract}
The idea of the present paper is to study orbital transfers for a spacecraft from the Lagrangian points in the Earth-Moon system to the Earth, as well as to the other Lagrangian points. The model used is the restricted three-body problem with the addition of the effects of the radiation pressure in the trajectory of the spacecraft. The basic maneuver is a bi-impulsive transfers under the dynamics explained before. The results show that the radiation pressure has an influence in the process, although not very large. After that we made some simulations for similar maneuvers in a double asteroid system, where the gravitational forces are smaller and the radiation pressure gives important contributions to the motion of the spacecraft. It is possible to find solutions with smaller fuel consumption when considering the solar radiation pressure, in both cases, but with important reductions in the magnitude of the impulses required for the asteroid system. It is important always to take into account that the idea presented here is not to use the radiation pressure as a control, but just to measure its effects when performing the bi-impulsive transfer.
\end{abstract}

\footnotetext{
${ }^{1}$ Doctoral student, National Institute for Space Research - INPE, São José dos Campos, Brazil and Professor at Federal Center for Technological Education of Minas Gerais - CEFET-MG, Contagem, Brazil. magela@contagem.cefetmg.br. AIAA Member.

${ }^{2}$ President of the Board of the Graduate School at INPE in Brazil, National Institute for Space Research - INPE, São José dos Campos, Brazil. antonio.prado@inpe.br. AIAA Associate Fellow.

${ }^{3}$ Postdoctoral fellow, DMC, National Institute for Space Research - INPE, São José dos Campos, Brazil. diogo.sanchez@inpe.br / School of Aeronautics and Astronautics, Purdue University, 701 West Stadium Avenue, West Lafayette, IN 47907-2045, USA. dmerguiz@purdue.edu

${ }^{4}$ Professor at São Paulo State University, FEG/UNESP, Guaratinguetá, Brazil. vivian.gomes@ @eg.unesp.br 


\section{Introduction}

The equilibrium Lagrangian points that appear in the restricted three-body problem ${ }^{1}$ have several applications, like the location of space stations, relay satellites for communications, etc. ${ }^{2,3}$ They are five points of equilibrium of the system and a spacecraft placed there with zero velocity will remain there forever. $\mathrm{L}_{1}, \mathrm{~L}_{2}$ and $\mathrm{L}_{3}$ are the collinear points, located in the line connecting the two primaries, and they are always unstable. $\mathrm{L}_{4}$ and $\mathrm{L}_{5}$ are the triangular points, because they make an equilateral triangle with the two primaries. They are stable for the more important cases of the Solar System (Earth-Moon, Sun-Earth, Sun-Jupiter). Figure 1 shows a sketch of the locations of those points.

The present paper considers the problem of bi-impulsive transfers between the Lagrangian points and the Earth, in the Earth-Moon system, as well as from one Lagrangian point to another. After that, a double asteroid system ${ }^{4,5}$ is also used for the simulations. The main goal is to show the relative importance of the radiation pressure as a function of the masses of the primaries. It will be shown that this point is very important and the effects of the radiation pressure goes from almost negligible to very high, depending on the system of primaries. It is considered that the maneuver starts with the application of the first impulse at the initial position of the spacecraft and ends with the application of the second impulse in the final position of the spacecraft. The effects of the radiation pressure is not used as a control, but it is assumed to be a perturbation present in the system. Previous researches have been done in similar problems. ${ }^{6,7,9}$

The effects of the solar radiation pressure in the trajectory of the spacecraft can be modulated by changing the area/mass of the spacecraft, so it is possible to increase those effects by adding large panels to the spacecraft, if it is interesting for the mission. Those panels can also be used to get solar energy to supply the spacecraft, so they do not have to be done only for this maneuver.

\section{Mathematical model}

The planar circular restricted three-body problem with the addition of the solar radiation pressure is used as the mathematical model. It is assumed that two bodies $\mathrm{M}_{1}$ and $\mathrm{M}_{2}$ are orbiting their common center of mass in circular Keplerian orbits and a third body $\mathrm{M}_{3}$, with negligible mass, is orbiting these two main bodies, called primaries. The motion of the third body, $\mathrm{M}_{3}$, is affected by the two main bodies $\mathrm{M}_{1}$ and $\mathrm{M}_{2}$, but it does not affect their motion. ${ }^{1}$ To represent the motion of the bodies, the canonical system of units is used. This system implies that:

1) The unit of distance is the distance between the primaries;

2) The angular velocity of the motion of $\mathrm{M}_{1}$ and $\mathrm{M}_{2}$ is assumed to be one;

3) The mass of the smaller primary $\left(M_{2}\right)$ is given by the mass ratio $\mu=\frac{M_{2}}{M_{1}+M_{2}}$, and the mass of the primary $M_{1}$ is given by $(1-\mu)$;

4) The unit of time is defined such that the period of the motion of the primaries is $2 \pi$;

5) The gravitational constant, G, is considered one.

The equations of motion of $\mathrm{M}_{3}$ (spacecraft) are given by:

$$
\begin{aligned}
& \ddot{x}-2 \dot{y}=\frac{\partial \Omega}{\partial x}+F_{X} \\
& \ddot{y}+2 \dot{x}=\frac{\partial \Omega}{\partial y}+F_{Y}
\end{aligned}
$$

where $\Omega$ is the pseudo-potential given by:

$$
\Omega=\frac{1}{2}\left(x^{2}+y^{2}\right)+\frac{(1-\mu)}{r_{1}}+\frac{\mu}{r_{2}}
$$

where: 


$$
\begin{gathered}
r_{1}{ }^{2}=(x+\mu)^{2}+y^{2} \\
r_{2}{ }^{2}=(x-1+\mu)^{2}+y^{2}
\end{gathered}
$$

and $\mathrm{F}_{\mathrm{X}}$ and $\mathrm{F}_{\mathrm{Y}}$ are the components of the solar radiation pressure. The magnitude of this force is given by:

$$
a_{\text {solar }}=(1+C r) P_{s} \frac{A_{s}}{m d^{2}} \hat{f}
$$

where $\mathrm{Cr}$ is the reflectivity of the surface, from 0 to 1 ; $\mathrm{P}_{\mathrm{S}}$ is the solar constant, equal to $1360 \mathrm{~W} / \mathrm{m}^{2}$ at one A.U. (Astronomical Unit, that is the Sun-Earth distance) divided by the speed of light; $A_{s}$ is the area of the spacecraft that is illuminated by the Sun; $m$ is the mass of the spacecraft; $d$ is the distance Sun-spacecraft in A.U. The unit vector $\hat{f}$ represents the direction of the force, that is assumed to be the Sun-spacecraft line.

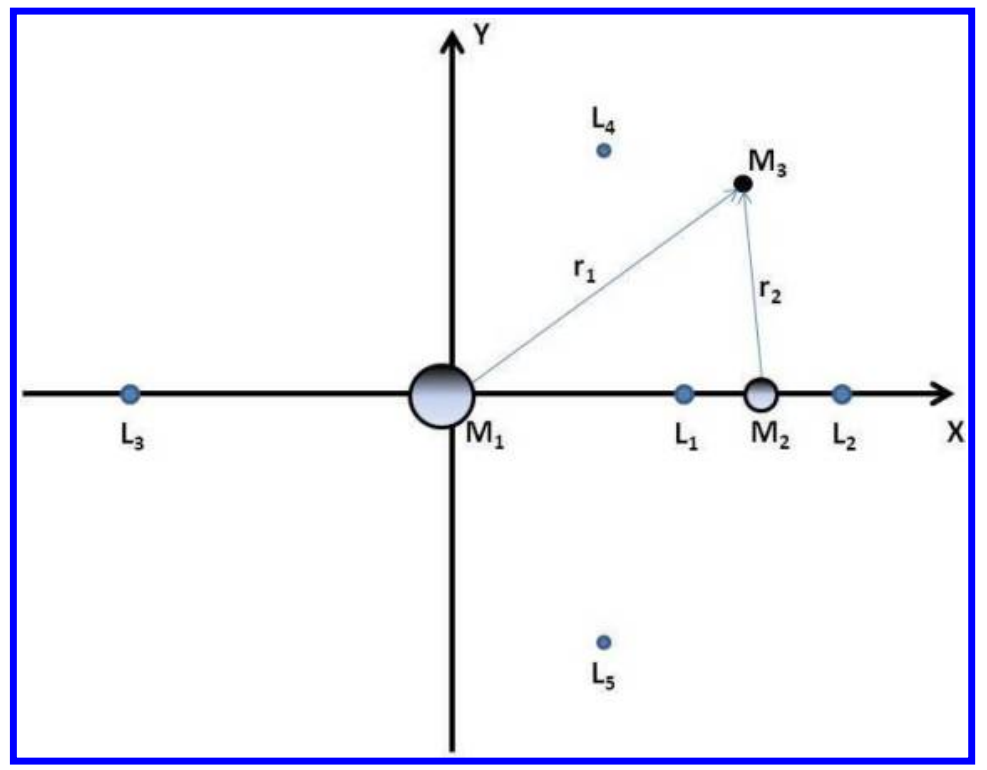

Figure 1. Location of the Lagrangian Points.

This system of equations has no analytical solutions, and numerical integration is required to solve the problem. The maneuvers are always assumed to be bi-impulsive, with the impulses applied at the beginning and at the end of the each transfer. The fuel consumed is specified by the total variation of velocity $(\Delta \mathrm{V})$ applied to the spacecraft in both impulses. To obtain solutions, the problem is treated as a TPBVP (Two Point Boundary Value Problem) and it is solved using numerical integrations and gradient methods. ${ }^{10}$

\section{Results}

The first families of transfer orbits studied considers transfers between the collinear Lagrangian points in the Earth-Moon system in two directions: clockwise and counterclockwise. The results are organized in plots of the $\Delta \mathrm{V}$ against the initial flight path angle (in degrees) in the rotating frame. Different locations of the Sun are used and shown in figure 2. Figures 3 to 6 show the results for the maneuvers among the three collinear Lagrangian points. They are plots showing the variation of velocity required by the transfers against the initial flight path angle. 


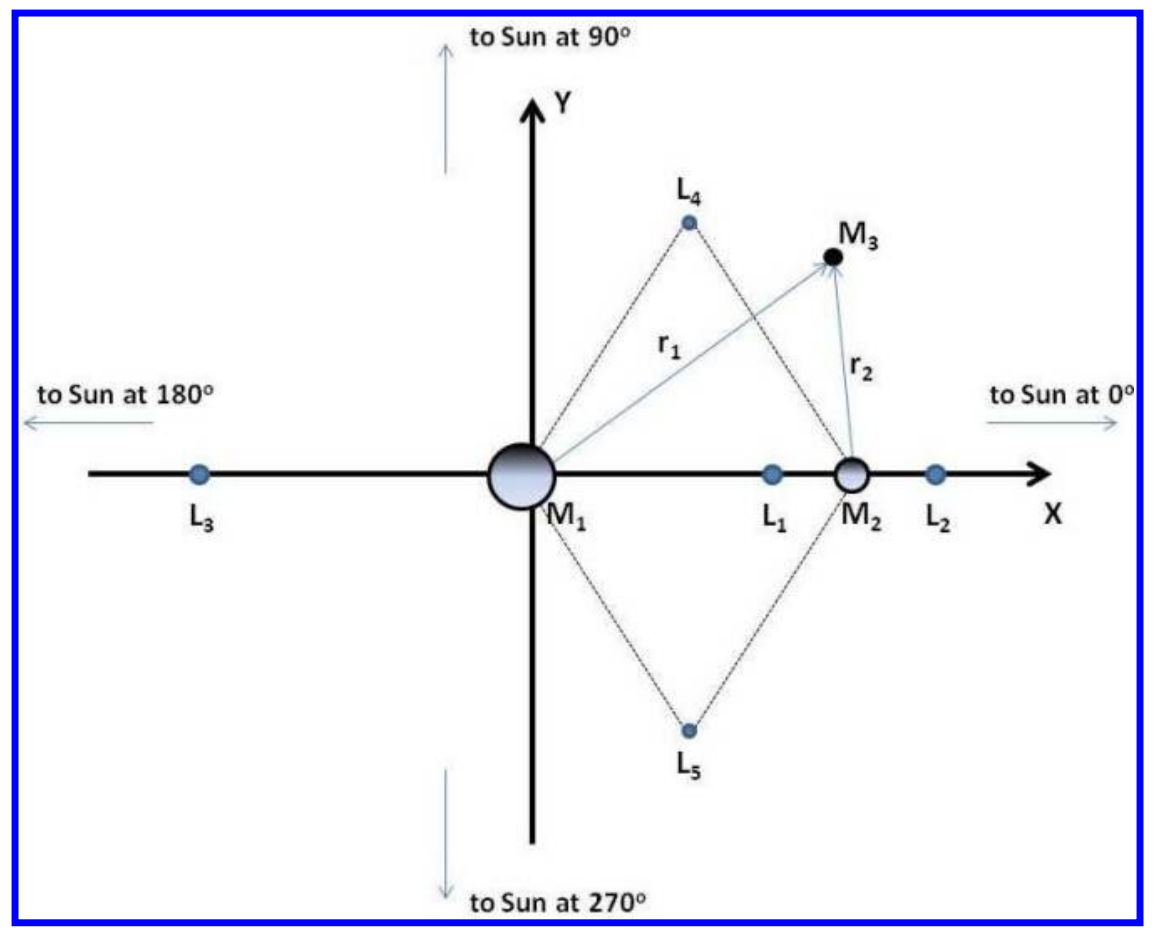

Figure 2. Location of the Sun relatively to the primaries.

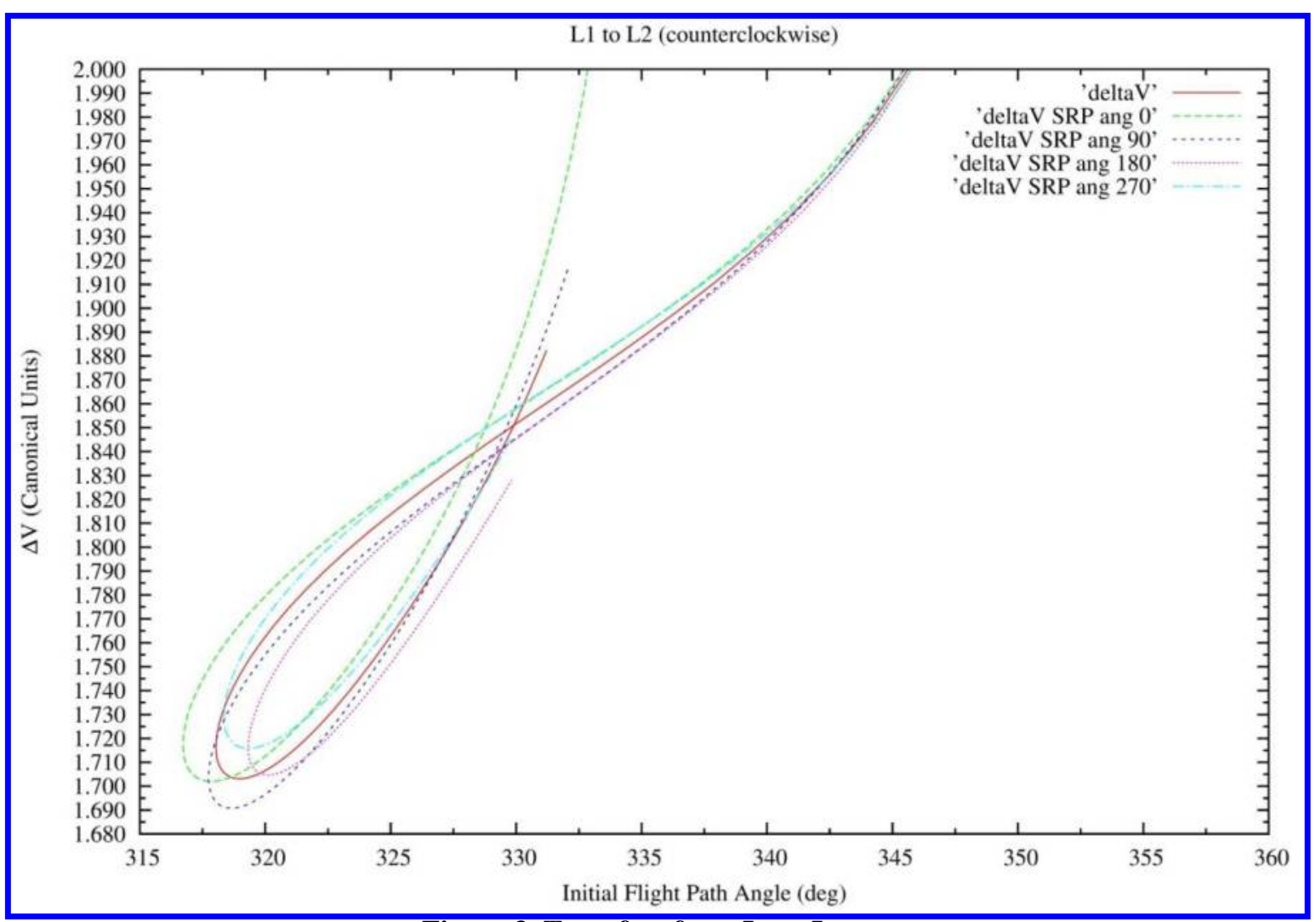

Figure 3. Transfers from $L_{1}$ to $L_{2}$.

4

American Institute of Aeronautics and Astronautics 


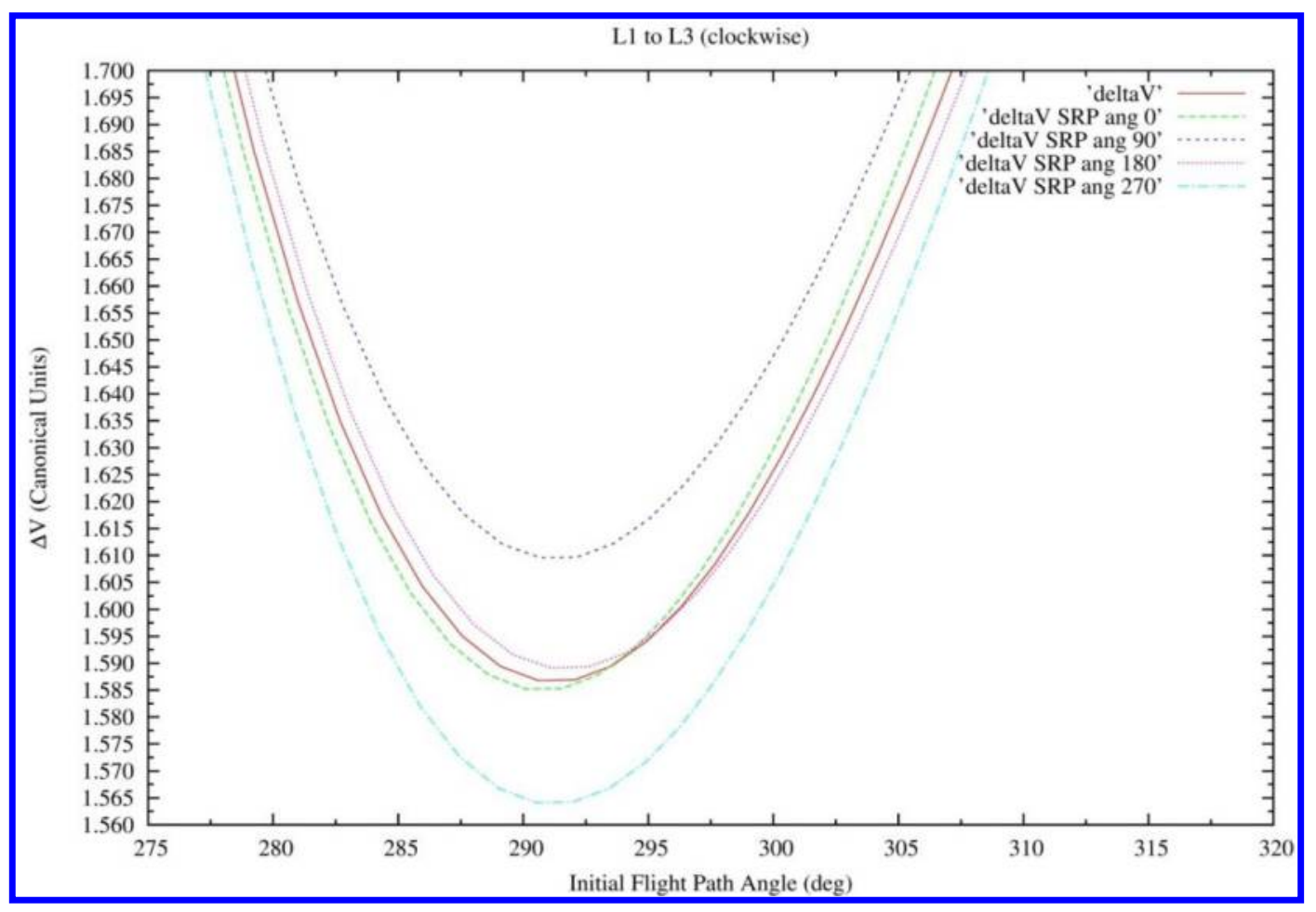

Figure 4. Transfers from $L_{1}$ to $L_{3}$.

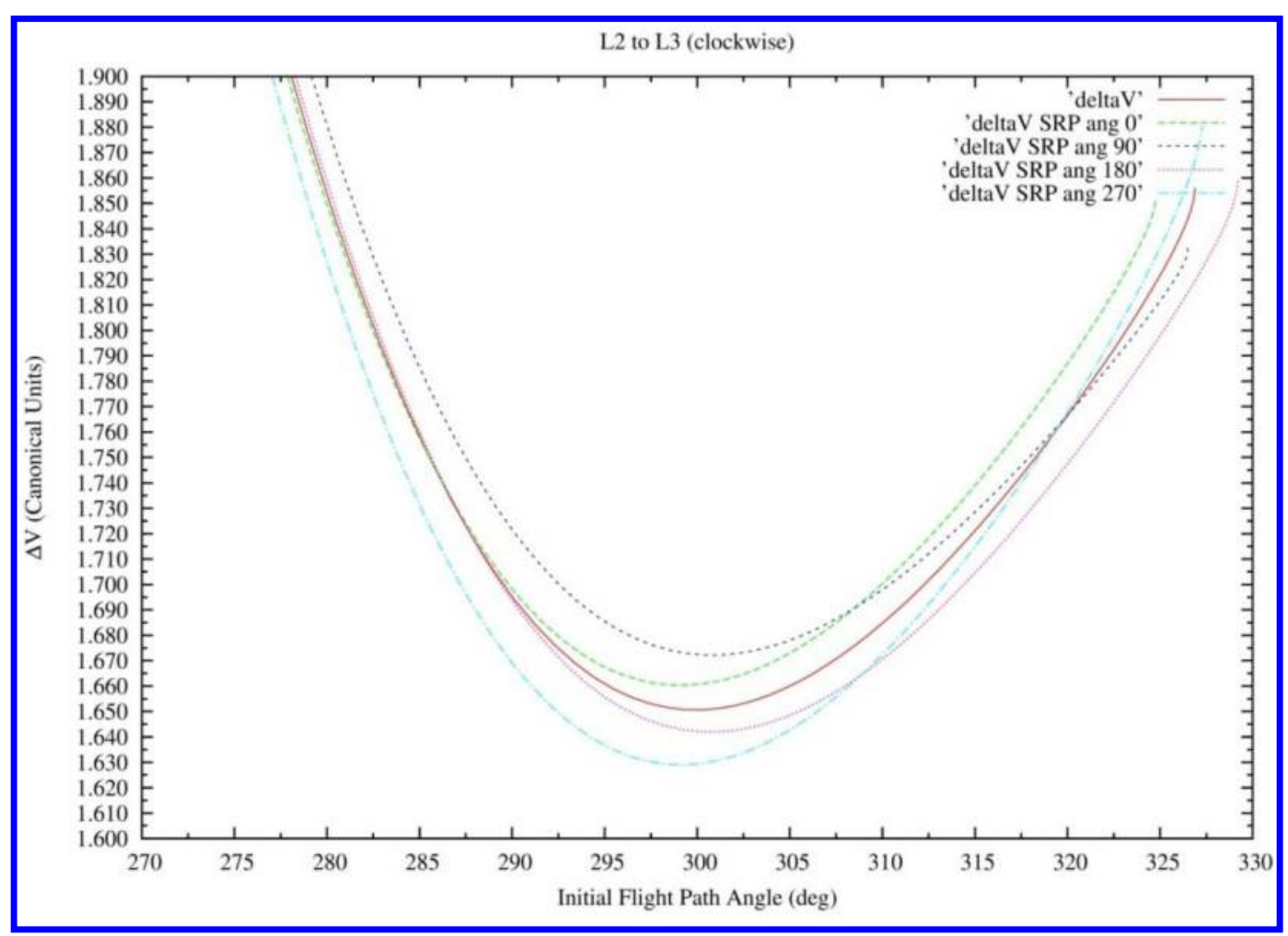

Figure 5. Transfers from $L_{2}$ to $L_{3}$. 5

American Institute of Aeronautics and Astronautics 


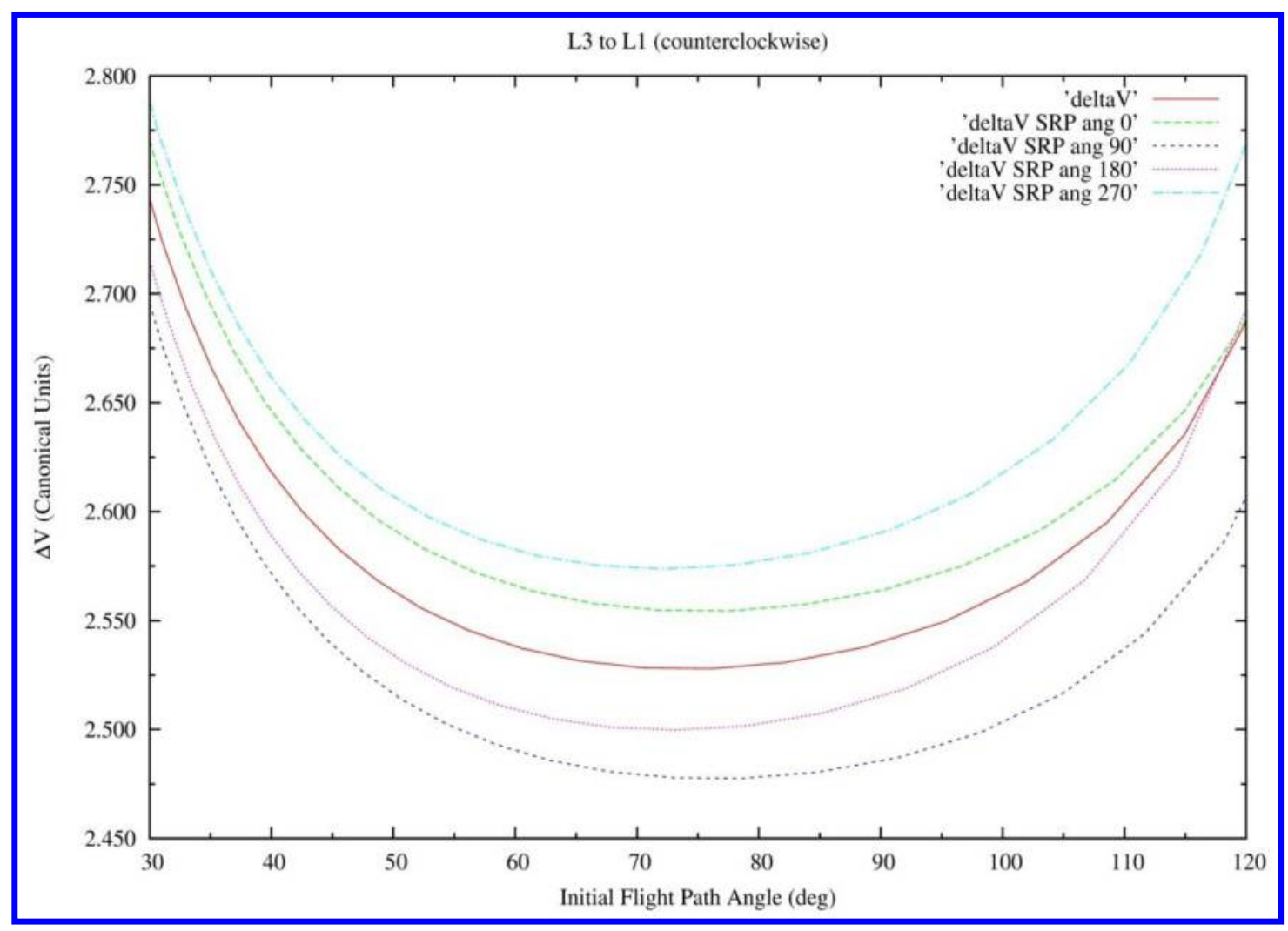

Figure 6. Transfers from $L_{3}$ to $L_{1}$.

The next study considers transfers between the Lagrangian points and the Earth, also using several values for the time of flight and two directions for the transfer: clockwise and counterclockwise. Figures 7 to 11 show the results, plotting the variation of velocity against the initial flight angle, as done before. 

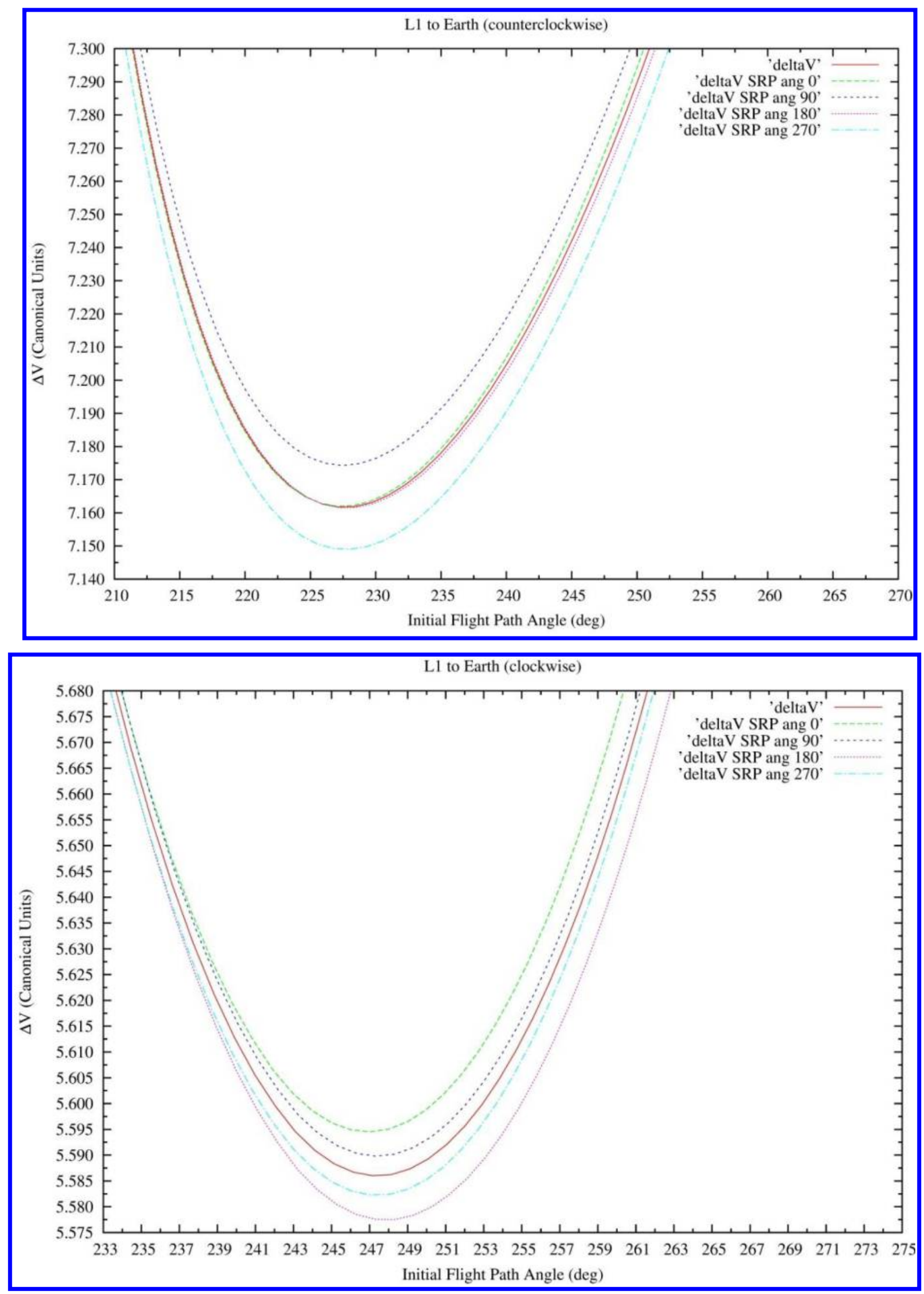

Figure 7. Transfers from $L_{1}$ to the Earth.

American Institute of Aeronautics and Astronautics 


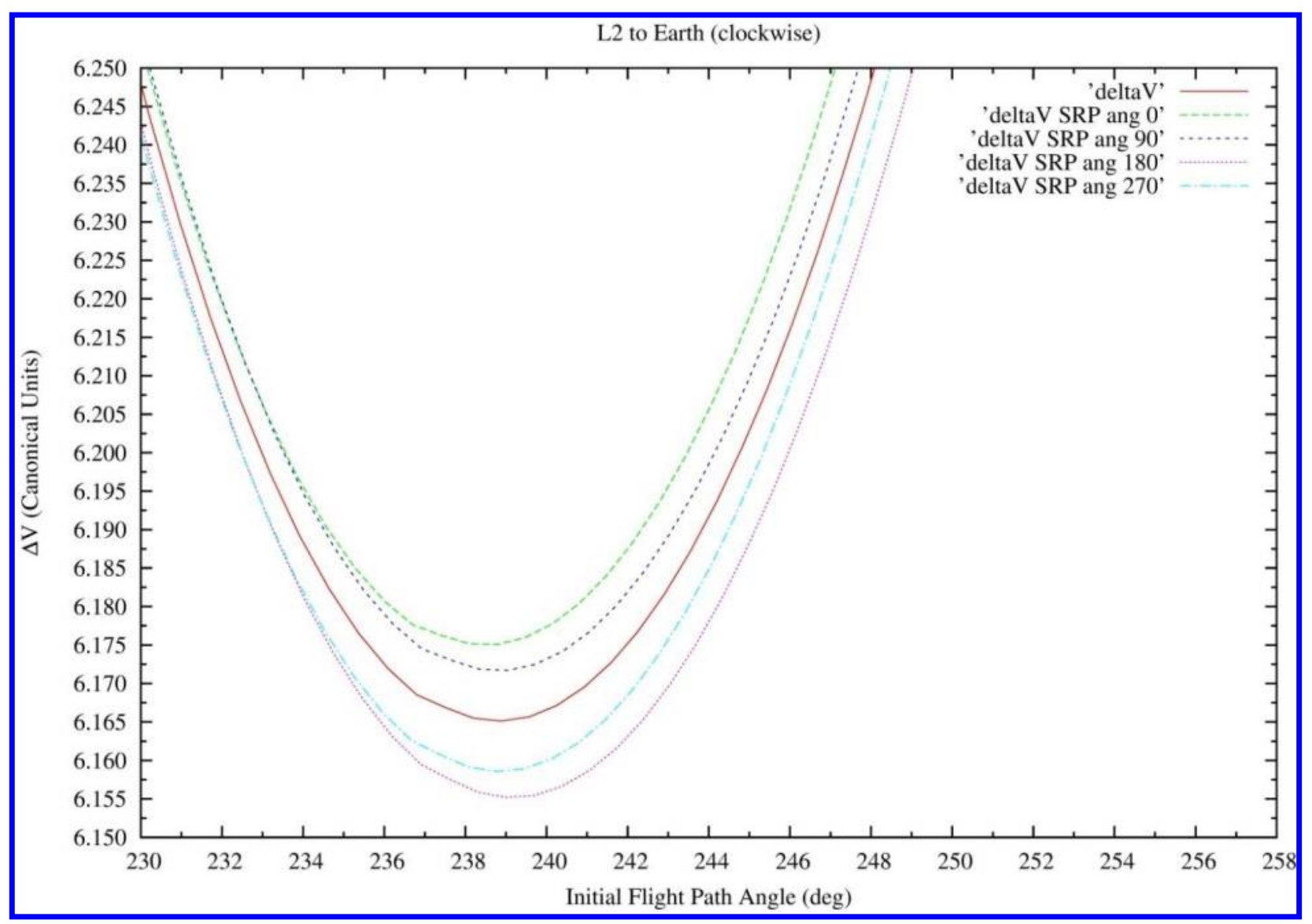

Figure 8. Transfers from $L_{2}$ to the Earth. 

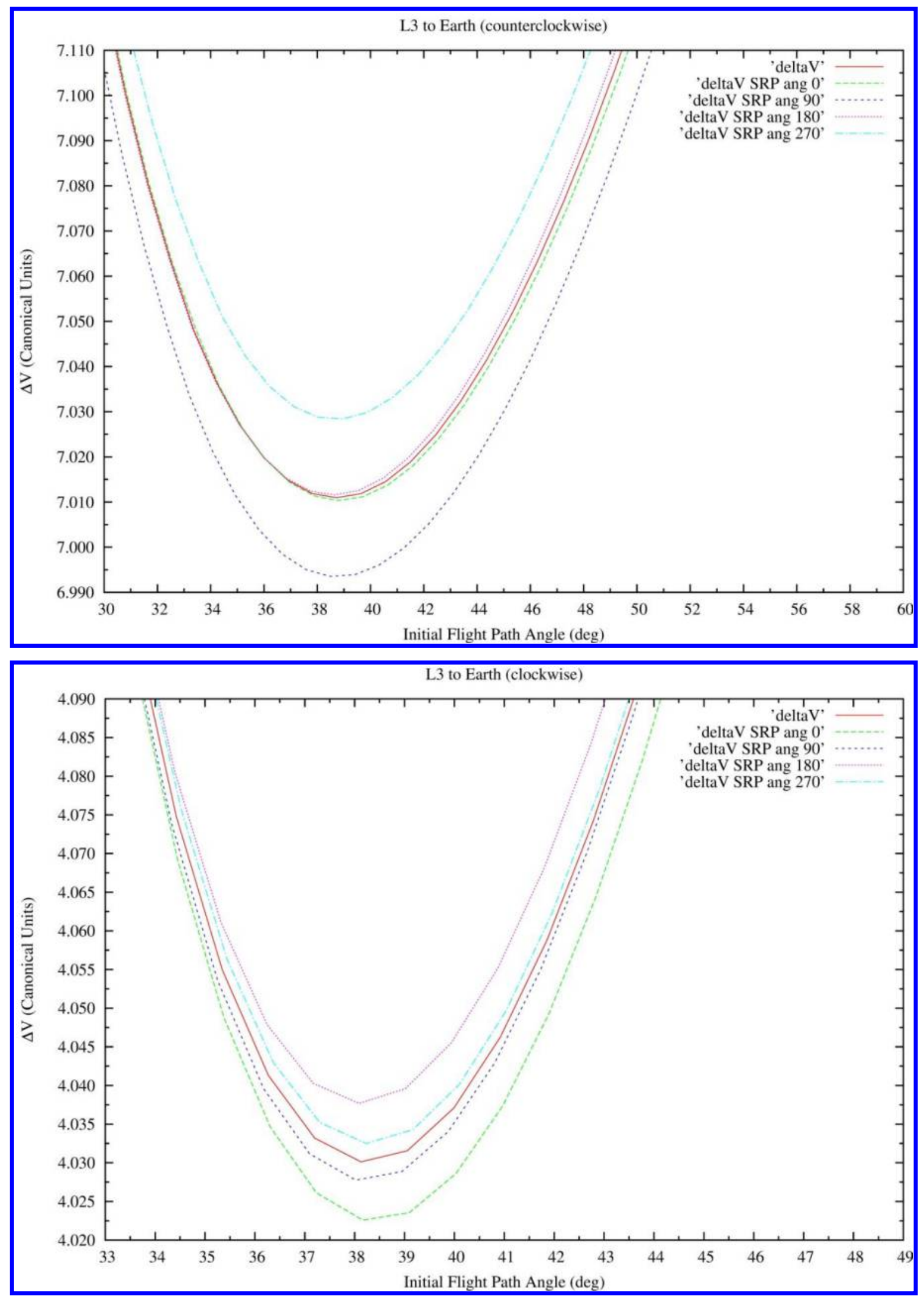

Figure 9. Transfers from $L_{3}$ to the Earth.

American Institute of Aeronautics and Astronautics 

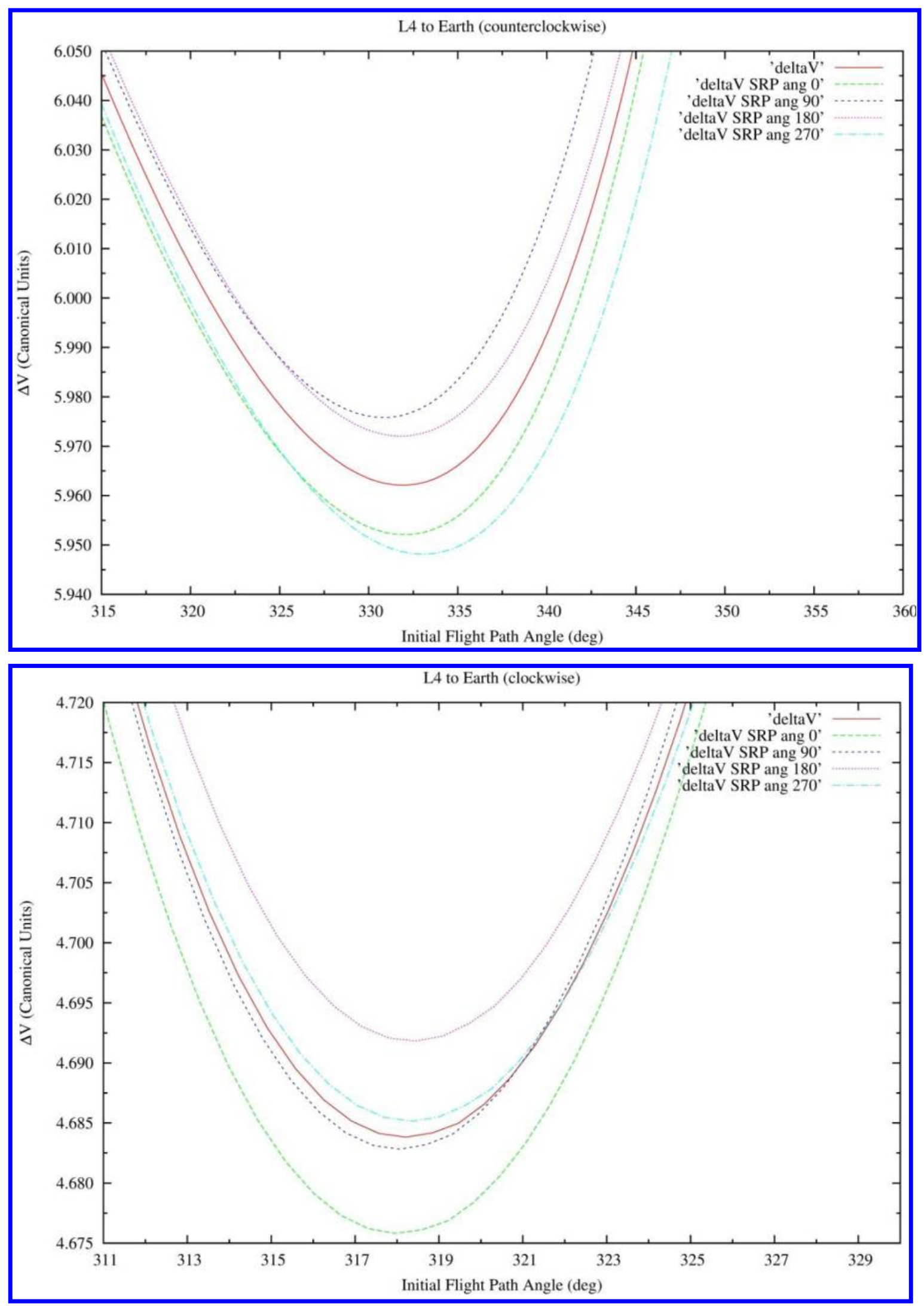

Figure 10. Transfers from $L_{4}$ to the Earth.

American Institute of Aeronautics and Astronautics 

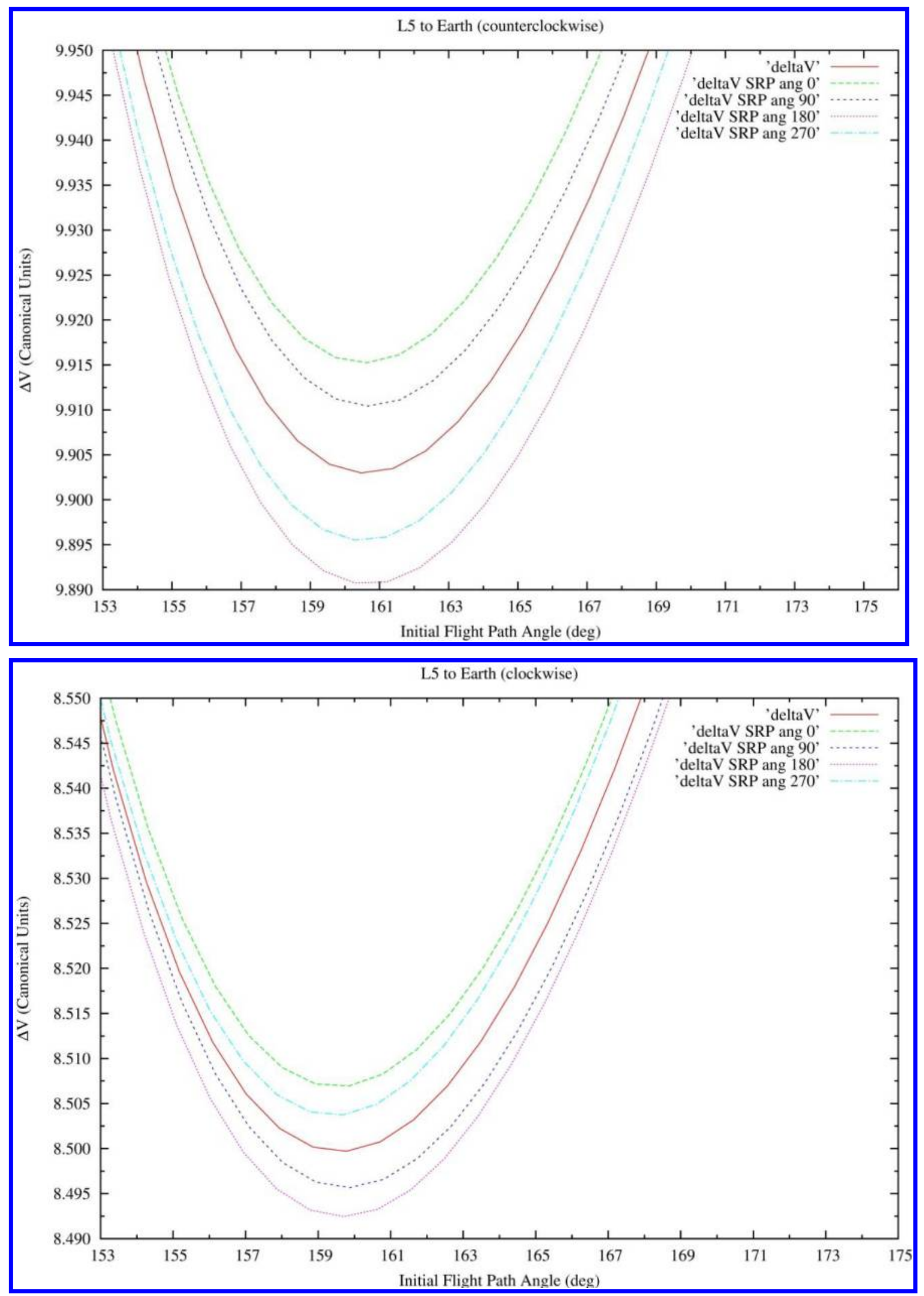

Figure 11. Transfers from $L_{5}$ to the Earth.

11

American Institute of Aeronautics and Astronautics 
The next session of results shows transfers in an asteroid system. The asteroid system used is the $2001 \mathrm{SN}_{263}{ }^{4,5}$, which is a triple system under study for the Aster mission. ${ }^{11}$ It is a triple system, but the full dynamics is not considered to calculate the Lagrangian points and the transfers itself. Instead, one double system with the larger body, asteroid Alpha, and one of the smaller ones, in the present case the asteroid Beta, is considered. The transfers considered are between the collinear Lagrangian points and the main asteroid, the asteroid Alpha. This system has an eccentricity $\mathrm{e}=0.48$ and a semi-major axis a $=1.99$ A.U. ${ }^{5}$. The distance considered for the simulations is the periapsis, which is about 1 A.U.

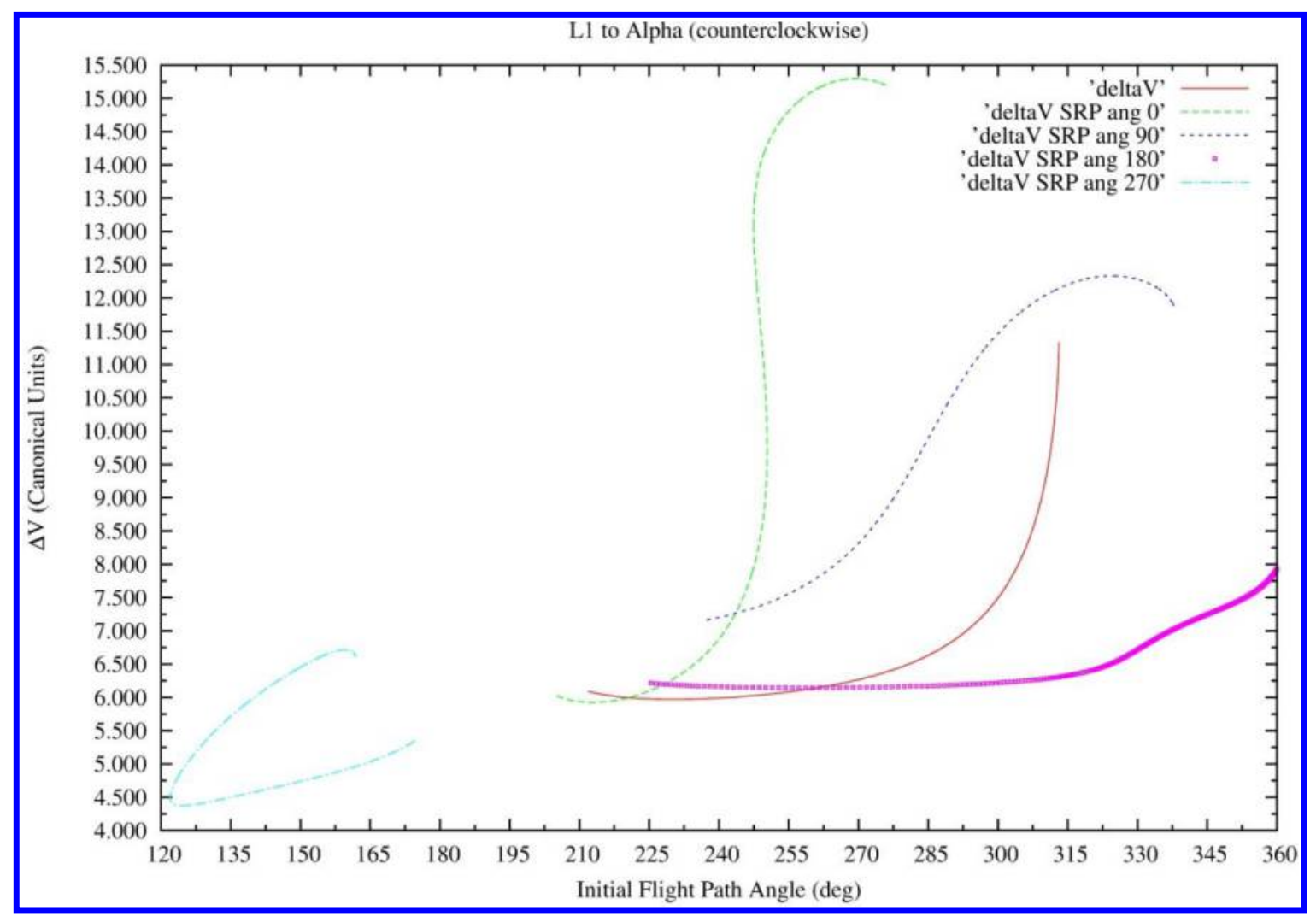




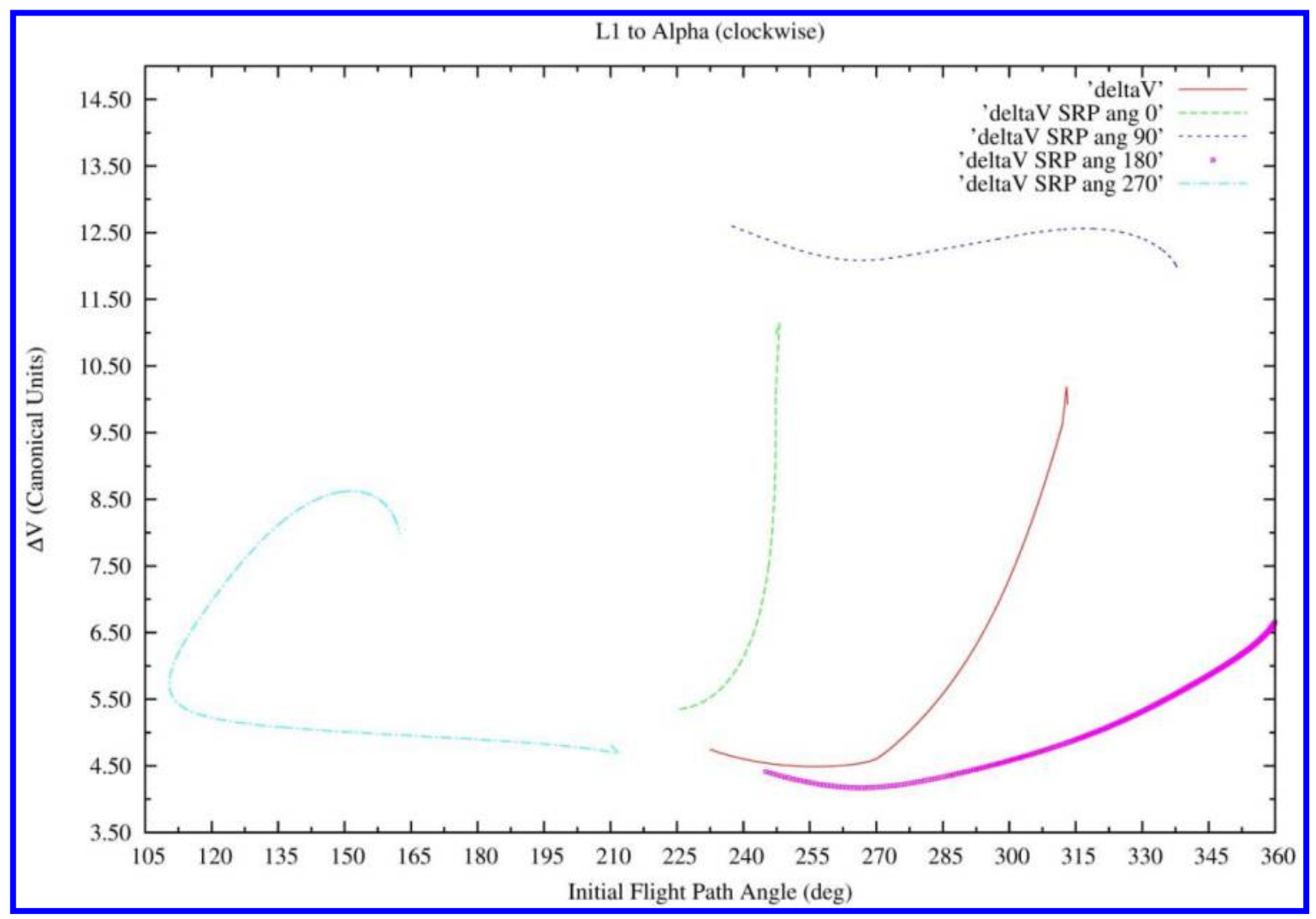

Figure 12. Transfers from $L_{1}$ to asteroid Alpha. 

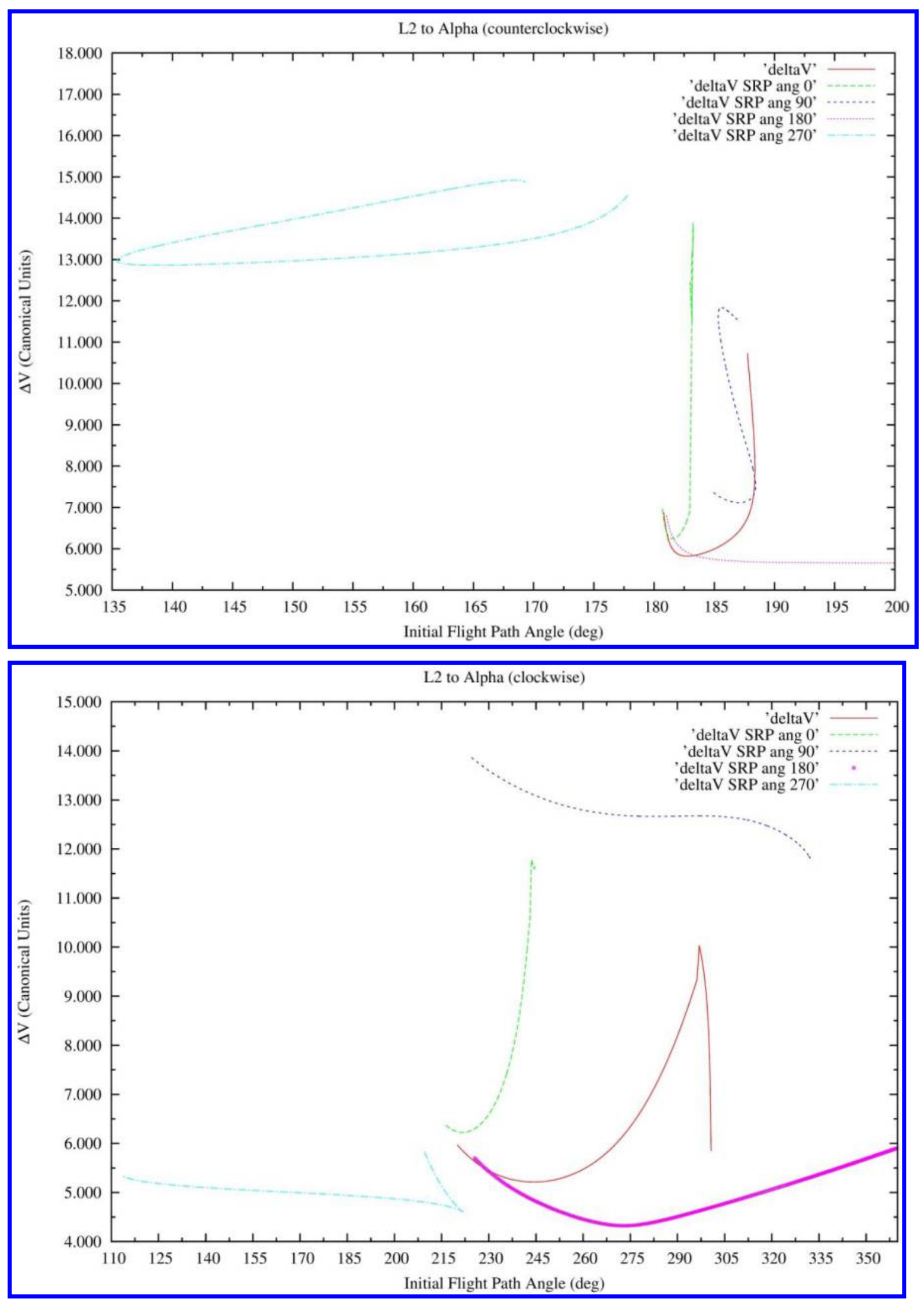

Figure 13. Transfers from $L_{2}$ to asteroid Alpha.

American Institute of Aeronautics and Astronautics 

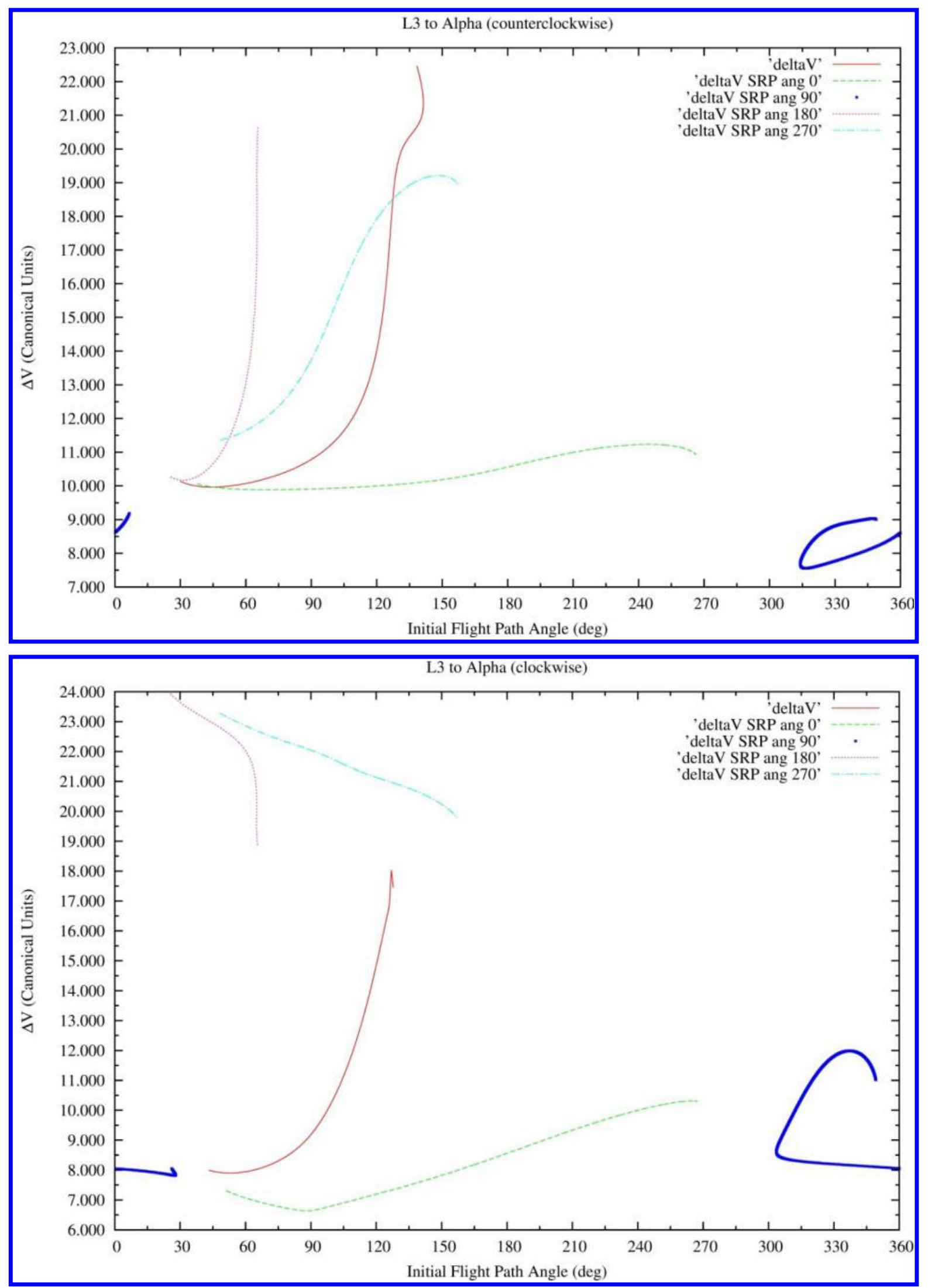

Figure 14. Transfers from $L_{3}$ to asteroid Alpha.

15

American Institute of Aeronautics and Astronautics 


\section{Conclusions}

The effects of the radiation pressure in a bi-impulsive transfer between the Lagrangian points and from the Lagrangian points to the Earth were studied in the Earth-Moon system.

The results indicated that the radiation pressure modifies the trajectory of the spacecraft, modifying the energy required for the transfers. It is possible to choose the right moment to perform the maneuver such that the magnitudes of the impulses to be applied are minimized. So, the moment to start the maneuver is a type of indirect control. For the Earth-moon system the differences are small, due to the large gravity of the bodies involved.

When considering smaller bodies, like the asteroid system, the importance of the radiation pressure increases, and the locations and values of the best transfers are different. It is also possible to choose the right moment to perform the maneuver, such that the magnitudes of the impulses to be applied are minimized. The difference is that, in those cases, the savings are very large.

\section{Acknowledgments}

The authors wish to express their appreciation for the support provided by grants \#2011/13101-4, 2011/08171-3, 2012/21023-6, 2014/22295-5 and 2016/14665-2, from São Paulo Research Foundation (FAPESP), grants \#301338/2016-7, 4068411/2016-0 and 473164/2013-2 from the National Council for Scientific and Technological Development (CNPq).

\section{References}

${ }^{1}$ Szebehely, V., Theory of Orbits, Academic Press, New York (1967).

${ }^{2}$ Bond, V. R., Sponaugle, S. J., Fraietta, M. F. and Everett, S. F., Cislunar libration point as a transportation node for lunar exploration. AAS Paper 91-103, AA.S/AlAA Spaceflight Mechanics Meeting, Houston, Texas (1991).

${ }^{3}$ Farqhar, R. W., Future missions for libration-point satellites. Astronautics and Aeronautics 5, 52-56 (1969).

${ }^{4}$ Araujo, R.A.N., Winter, O.C., Prado, A.F.B.A., Stable retrograde orbits around the triple system 2001 SN263. Monthly Notices of the Royal Astronomical Society, 449(4), 4404-4414, 2015.

${ }^{5}$ Araujo, R.A.N., Winter, O.C., Prado, A.F.B.A., Sukhanov, A. Stability regions around the components of the triple system 2001 SN263. Monthly Notices of the Royal Astronomical Society, 423(4), 3058-3073, 2012.

${ }^{6}$ Broucke, R., Traveling Between the Lagrange Points and the Moon, Journal of Guidance and Control, vol. 2, n. 4, pp. 257-263, 1979.

${ }^{7}$ Cabette, R.E.S., Prado, A.F.B.A., Transfer orbits to/from the Lagrangian points in the restricted four-body problem. Acta Astronautica,63(11), 1221-1232, 2008.

${ }^{8}$ Prado, A.F.B.A., Orbital maneuvers between the Lagrangian points and the primaries in the Earth-Sun system. Journal of the Brazilian Society of Mechanical Sciences and Engineering, 28(2), 131-139, 2006.

${ }^{9}$ Prado, A.F.B.A., Traveling between the Lagrangian points and the Earth. Acta Astronautica, v. 39, n.7, p. 483-486, 1996.

${ }^{10}$ Press, W. H., Flannery, B. P., Teukolsky, S. A. and Vetterling, W. T., Numerical Recipes, Cambridge University Press, NewYork, 1989.

${ }^{11}$ Sukhanov, A., Velho, H.F.C., Macau, E., Winter, O.C., The Aster Project: Flight to a Near-Earth Asteroid, Cosm. Res., 48(5), 443-450, 2010. 